\title{
Patrick Rössler; Klaus Kamps; Gerhard Vowe: Weimar 1924: Wie Bauhauskünstler die Massenmedien sahen. Die Meistermappe zum Geburtstag von Walter Gropius
}

Stuttgart: Franz Steiner Verlag 2019. 208 Seiten. Preis: $€ 19,90$

\section{Juliana Raupp}

Online publiziert: 11. März 2020

(C) Der/die Autor(en) 2020

Am Anfang steht ein Foto: Darauf zu sehen ist eine Menschenmasse, die, von schräg oben von einem Fenster aus aufgenommen, von einem großen, altmodisch anmutenden Lautsprecher beschallt wird. Das Foto stammt von dem Pressefotografen John Graudenz und zeigt die Bekanntgabe der Wahlergebnisse zur Reichstagswahl im Mai 1924 als erstes public listening-Event in Deutschland. Dieses Foto nahm der Bauhausmeister László Moholy-Nagy zum Anlass, um seinen Bauhauskollegen, den Meistern Lyonel Feininger, Wassili Kandinsky, Paul Klee, Georg Muche und Oskar Schlemmer eine Aufgabe zu stellen: Sie sollten das Foto künstlerisch interpretieren, so dass am Ende sechs Variationen der Vorlage von allen Bauhausmeistern vorliegen. Der Zeitungsausschnitt mit dem Foto und die sechs Variationen fasste MoholyNagy in einer Mappe zusammen, die dem Gründer des Bauhauses, Walter Gropius, zu dessen 41. Geburtstag am 17. Mai 1924 als Geschenk überreicht wurde.

Diese Geburtstagsmappe steht am Anfang des hier zu rezensierenden Buches: So wie Moholy-Nagy seinen Kollegen die Aufgabe stellte, das Foto künstlerisch zu interpretieren, stellten sich die drei Kommunikationswissenschaftler Patrick Rössler, Klaus Kamps und Gerhard Vowe die Aufgabe, das Foto sowie die einzelnen Blätter der Bauhausmeister kommunikationswissenschaftlich zu interpretieren. Und gab das Pressefoto von Graudenz für die Bauhausmeister ein zeitgenössisches Motiv ab, das es mit künstlerischen Mitteln zu interpretieren galt, so stellt es in den Augen der Kommunikationswissenschaftler, die aus heutiger Sicht darauf blicken, ein Dokument des Medienwandels dar: Sie übersetzen die Pressefotografie ebenso wie die

Prof. Dr. J. Raupp ( $\bowtie)$

Institut für Publizistik- und Kommunikationswissenschaft, Freie Universität Berlin,

Garystr. 55, 14195 Berlin, Deutschland

E-Mail: juliana.raupp@fu-berlin.de 
künstlerischen Variationen der Bauhausmeister in akademische Reflexionen über die Verbindung von Kunst-, Sozial- und Kommunikationsgeschichte.

Jeder der drei Kollegen nimmt dabei eine andere Perspektive ein: Rössler nähert sich werkorientiert und deutet die jeweiligen Motive inhaltlich und ästhetisch. Dabei stellt er Bezüge zum künstlerischen Schaffen und auch zu den unterschiedlichen Persönlichkeiten der jeweiligen Bauhausmeister dar: Oskar Schlemmer entpuppt sich in seinen Augen als eher humorloser Akademiker, Moholy-Nagy als technikbegeisterter Innovator, Klee als verspielter Visionär. Im Werk von Georg Muche erkennt Rössler ein Vexierbild, das er als ambivalente Auseinandersetzung mit der Publikumsrolle deutet. Kandinskys Beitrag stellt sich ihm als synästhetisches Bild eines reifen Künstlers dar, wogegen Feiningers zartes Aquarell bildlich und programmatisch aus der Reihe fällt und ihn als Träumer ausweist.

Die zeitdiagnostischen Einlassungen von Klaus Kamps kontextualisieren die Blätter und verweben diese mit politik- und sozialgeschichtlichen Details. Damit entfernt sich Kamps von den einzelnen Werken, bietet aber gerade dafür Wissenswertes über die Kommunikationsgeschichte in der Weimarer Republik. Themen sind die Wahlkampfkommunikation, die Geschichte des Kinos, die Rolle von Sport und Freizeitvergnügungen, die sozialen und politischen Milieus und Verwerfungen in der Weimarer Republik sowie die Kontraste zwischen Wirtschaftswachstum, Innovation und Inflation.

Gerhard Vowe schließlich stellt kommunikationstheoretische Fragen an jedes einzelne Bild in der Mappe und löst die Aufgabe damit besonders kreativ. Paul Klee wird zum Anlass genommen, um über die Rolle des Kommunikators und die Möglichkeiten persuasiver Kommunikation nachzudenken, Muches Arbeit motiviert ihn, über die Fragmentierung des Publikums nachzudenken, Kandinskys Werk, um Wirkungszusammenhänge zu überdenken. Und so weit, wie sich Feininger vom Pressefoto von Graudenz entfernte, entfernt sich Vowe von Feiningers Bild, wenn er über Mobilkommunikation schreibt und en passant verfestigte Sichtweisen in der Kommunikationswissenschaft hinterfragt.

Es ist dem Band anzumerken, dass die drei Verfasser sichtlich Spaß am gemeinsamen Projekt hatten. Die grenzüberschreitende Auseinandersetzung mit Kunstwerken, die in unserem Fach nicht gerade üblich ist, löst beim Lesen der Texte und beim Betrachten der Bilder und Fotografien in jedem Fall Inspiration und interessante Assoziationen aus. „Auch Forscher brauchen Phantasie!“" ruft Vowe (S. 182) aus. Und in der Tat ist phantasievoll, wie er zum Beispiel das pflanzenähnliche Element, das am unteren Bildrand des Werks von Paul Klee zu sehen ist und das in Vowes Augen „mir nichts, dir nichts“ (S. 124) erscheint, als unintendierten Effekt deutet.

Man nimmt das Buch gerne zur Hand - eine Seltenheit in Zeiten, in denen fast nur noch E-Books und PDFs gelesen werden. Erstaunlich ist die Ähnlichkeit zwischen sozial- und kommunikationswissenschaftlichen Abbildungen - der grafischen Darstellung des Gatekeeper-Modells, der Esser'schen Badewanne oder der Visualisierung von Filter Bubbles - und den abstrakten Motiven der Bauhausmeister, die in dem Band sichtbar gemacht wird. Und so freut man sich über das dezidiert kommunikationswissenschaftliche Geschenk zum 100. Geburtstag des Bauhauses im Jahr 2019. Das Buch ist zweisprachig in Deutsch und Englisch, reich bebildert, und zum Schluss gibt es noch ein Dessert: Der Fotokünstler Ralf Kaspers wurde von den drei 
Autoren gebeten, das Pressefoto von Graudenz aus heutiger Sicht zu interpretieren - zu sehen ist ein digitaler Datenstrom.

Funding Open Access funding provided by Projekt DEAL.

Open Access Dieser Artikel wird unter der Creative Commons Namensnennung 4.0 International Lizenz veröffentlicht, welche die Nutzung, Vervielfältigung, Bearbeitung, Verbreitung und Wiedergabe in jeglichem Medium und Format erlaubt, sofern Sie den/die ursprünglichen Autor(en) und die Quelle ordnungsgemäß nennen, einen Link zur Creative Commons Lizenz beifügen und angeben, ob Änderungen vorgenommen wurden.

Die in diesem Artikel enthaltenen Bilder und sonstiges Drittmaterial unterliegen ebenfalls der genannten Creative Commons Lizenz, sofern sich aus der Abbildungslegende nichts anderes ergibt. Sofern das betreffende Material nicht unter der genannten Creative Commons Lizenz steht und die betreffende Handlung nicht nach gesetzlichen Vorschriften erlaubt ist, ist für die oben aufgeführten Weiterverwendungen des Materials die Einwilligung des jeweiligen Rechteinhabers einzuholen.

Weitere Details zur Lizenz entnehmen Sie bitte der Lizenzinformation auf http://creativecommons.org/ licenses/by/4.0/deed.de.

Dr. Juliana Raupp ist Professorin am Institut für Publizistik- und Kommunikationswissenschaft der Freien Universität Berlin. 